\title{
4-REGULAR AND SELF-DUAL ANALOGS OF FULLERENES
}

\author{
MATHIEU DUTOUR SIKIRIĆ AND MICHEL DEZA
}

\begin{abstract}
An $i$-hedrite is a 4-regular plane graph with faces of size 2, 3 and 4. We do a short survey of their known properties DeDuSh03, DeHuLi02, DeSt03 and explain some new algorithms that allow their efficient enumeration. Using this we give the symmetry groups of all $i$-hedrites and the minimal representative for each. We also review the link of 4-hedrites with knot theory and the classification of 4-hedrites with simple central circuits.

An $i$-self-hedrite is a self-dual plane graph with faces and vertices of size/degree 2, 3 and 4 . We give a new efficient algorithm for enumerating them based on $i$-hedrites. We give a classification of their possible symmetry groups and a classification of 4-selfhedrites of symmetry $T, T_{d}$ in terms of the Goldberg-Coxeter construction. Then we give a method for enumerating 4-self-hedrites with simple zigzags.
\end{abstract}

\section{INTRODUCTION}

A fullerene is a 3-regular plane graph whose faces have size 5 or 6. As a consequence of Euler's formula any fullerene has exactly 12 5 -gonal faces. For a 3-regular plane graph $G$ and a $r$-gonal face $F$ of $G$, the quantity $6-r$ is called curvature and Euler's formula is then a statement about the curvature on the sphere. A natural generalization of fullerene is the class of 3-regular plane graphs with faces of size between 3 and 6 (see, for example, [DeDuFo09]).

Here we consider another generalization, that is a suitable $k$-regular plane graph. The Euler formula $V-E+F=2$ becomes then

$$
\sum_{j=2}^{\infty} p_{j}(s-j)=\frac{4 k}{k-2} \text { with } s=\frac{2 k}{k-2} ;
$$

we will permit 2 -gons (doubled edges) but not 1 -gons. The only integral pairs $(s, k)$ are $(6,3),(4,4)$ and $(3,6)$. We will permit only $s$ - and $(s-1)$-gonal faces. So, $p_{s-1}=\frac{4 k}{k-2}$ and $p_{s}$ is not bounded. The number $n$ of vertices is

$$
n=\frac{4(k+2)}{(k-2)^{2}}+p_{s} \frac{2}{k-2} .
$$


For $k=3,4$ and 6 we get spherical analogs of the regular partition of the Euclidean plane $E^{2}:\left\{6^{3}\right\},\left\{4^{4}\right\}$ and $\left\{3^{6}\right\}$, respectively, where 12 pentagons, 8 triangles and 6 doubled edges play role of "defects", disclinations needed to increase the curvature to the one of sphere $S^{2}$. The graphs with smallest number $n$ of vertices have only $(s-1)$-gons; they are Dodecahedron, Octahedron and Bundle 6 (2 vertices connected by 6 edges) for $k=3,4,6$, respectively. The case $k=3$ gives fullerenes. The case $k=4$, i.e., of 4-regular plane graphs with faces of size 3 or 4 , gives octahedrites treated in the foundational paper DeSt03. Let us call graphs in the remaining case $k=6$ (6-regular plane graphs with faces of size 2 or 3) bundelites. Thurston's work [Thu98] implies that fullerenes can be parametrized by 10 Eisenstein integers and the number of fullerenes with $n$ vertices grows as $n^{9}$; those results can be generalized to octahedrites and bundelites. The ring of definition for octahedrites, respectively bundelites, is the Gaussian, respectively Eisenstein integers. Those cases belong to two of 94 cases enumerated in Thu98].

We present here a short review of known facts about octahedrites as established in DeDuSh03, DeHuLi02, DeSt03 and present a few new facts and applications. We give the possible symmetry groups of octahedrites and the graphs of minimal vertex-sets realizing them. Then we show how octahedrites can be used for the enumeration of a more general $i$-hedrites, i.e. 4-regular plane graphs with faces of size 2 , 3 or 4 and $p_{2}+p_{3}=i$.

Then we consider central circuit partition of the edge-sets of octahedrites and the corresponding knot-theoretic notions, that is alternating knot, Borromean link, and equivalence.

A $i$-self-hedrite is a plane graph with vertices and faces of size 2, 3 or 4 that is isomorphic to its dual with $p_{2}+p_{3}=i$. Such graphs have $2 p_{2}+p_{3}=4$ and $i$-self-hedrites can be enumerated effectively by using $2 i$-hedrites with a method detailed below. We determine their possible symmetry groups and we list the minimal representatives for each of them. We characterize the 4-self-hedrites of symmetry $T$ or $T_{d}$ in terms of the Goldberg-Coxeter construction for octahedrites. Then we give a method based on $2 i$-hedrites for determining the $i$-self-hedrites with simple zigzags.

The computations of this paper were done using the GAP computer algebra system and the computer packages polyhedral, plangraph of the first author. The enumeration of octahedrites was done using the ENU program by O. Heidemeier [He98, BrHaHe03] and the program CaGe [BDDH97] was used for making the drawings. 


\section{Structural Properties}

A plane graph is a graph drawn on the plane with edges intersecting only at vertices. A graph $G$ is 3-connected if after removing any 2 vertices of $G$ the resulting graph is connected. A 3-polyhedron is a 3-dimensional polytope, its skeleton defines a 3-connected plane graph and it is known that this characterizes the skeleton of 3-polytopes. Furthermore [Ma71], a 3-connected plane graph $G$ can be represented as a skeleton of a 3-polytope $P$ such that any symmetry of $G$ is realized as a isometry of the polytope $P$. We refer to DeDu08 for more details on such questions.

It is proved in DeDuSh03 that any octahedrite is 3-connected which implies that its symmetry groups is realized as isometry of 3-space. Since those group have been classified long ago and are much used in chemistry, we can use the chemical nomenclature here (see, for a possible presentation, Du04]).

An octahedrite exists for any $n \geq 6$ except $n=7$ (see Gr67, page 282]). For a 4-regular graph with $p_{j}$ denoting the number of faces of size $j$, the classical Euler formula $V-E+F=2$ can be rewritten (see [DeDu08, Chapter 1], for the easy details) as

$$
\sum_{j=2}^{\infty}(4-j) p_{j}=8 .
$$

For octahedrites this directly implies $p_{3}=8$. Octahedron is the unique octahedrite with $n=6$.

Theorem 1. The only symmetry groups of octahedrites are: $C_{1}, C_{s}$, $C_{2}, C_{2 v}, C_{i}, C_{2 h}, S_{4}, D_{2}, D_{2 d}, D_{2 h}, D_{3}, D_{3 d}, D_{3 h}, D_{4}, D_{4 d}, D_{4 h}, O$, $O_{h}$. The minimal possible representative are given in Figure 1 .

The proof that the list of groups is complete is given in DeDuSh03, but the minimal possible representatives were not determined at the time. The method is first to go through the restrictions that vertex degree and face size impose. An $m$-fold axis of rotation has necessarily $m=4$ (passing though a face of size 4 or a vertex), $m=3$ (axis passing through a face of size 3 ), or $m=2$ (axis passing though an edge, a vertex of degree 4 or a face of size 4 ). Then the classification of point groups gives a list of possible candidates. Some candidates are excluded for reason of orbit size and other similar simple arguments. But some groups are excluded for a subtler reason: the existence of a symmetry implies another symmetry. For example a 3-, 4-fold axis of symmetry, i.e. $C_{3}, C_{4}$ implies actually at least $D_{3}, D_{4}$ for possible symmetry groups. See DeDuSh03] for details. 

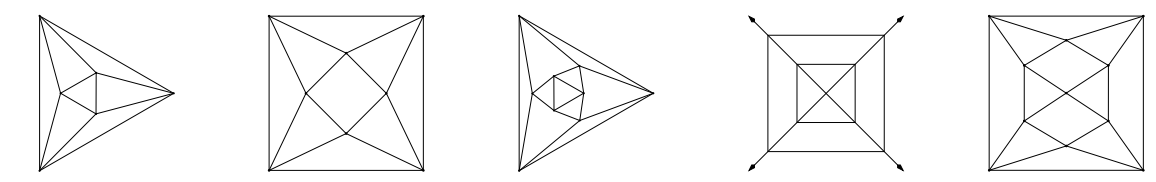

$O_{h}, n=6$
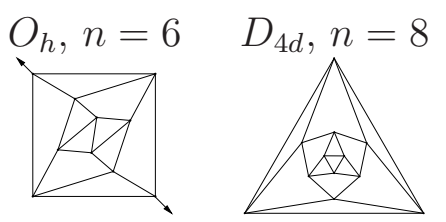

$D_{3 h}, n=9$

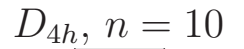

$C_{2 v}, n=11$
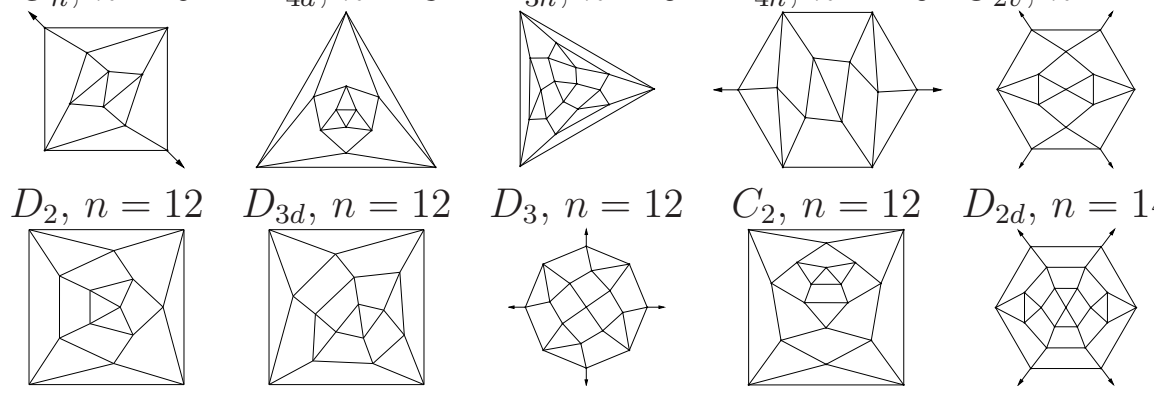

$D_{2 d}, n=14$
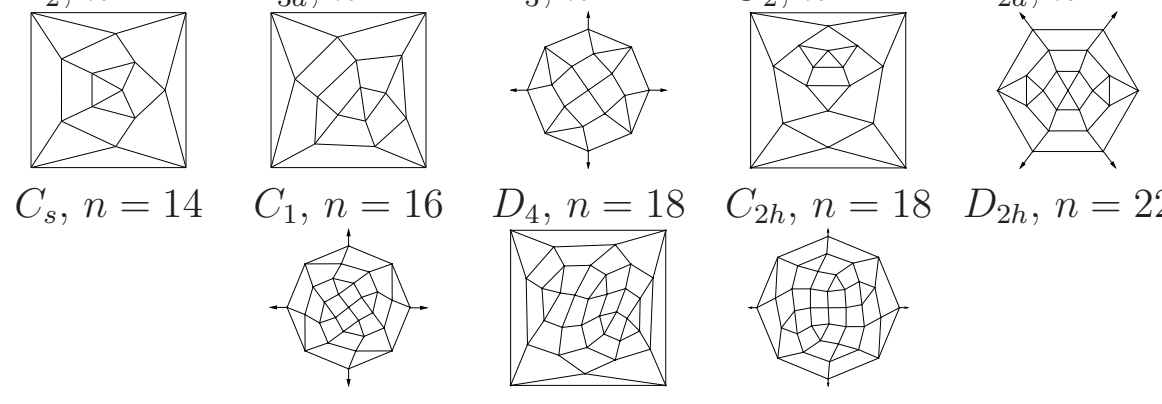

$D_{4}, n=18$

$C_{2 h}, n=18$

$D_{2 h}, n=22$

$O, n=30$
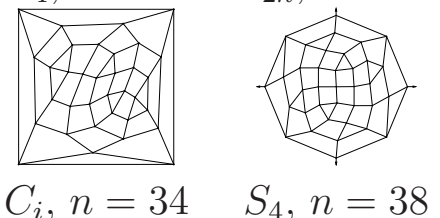

$S_{4}, n=38$

Figure 1. Minimal representatives for each possible symmetry group of an octahedrite

On the other hand, finding the minimal possible representative is done in a very non-clever way: we look at all the generated octahedrites and select the representatives with minimal vertex-sets. The enumeration of octahedrites was done by using the program ENU (see [He98, BrHaHe03]) by O. Heidemeier, that enumerates classes of 4regular graphs with constraint on the size of their faces, fairly efficiently.

\section{Generation of $i$-Hedrites}

Define an $i$-hedrite to be a 4-regular $n$-vertex plane graph, whose faces have size 2,3 and 4 only and $p_{2}+p_{3}=i$ (see, for more details, [DeDuSh03]). Using Formula (10), we get for an $i$-hedrite $2 p_{2}+p_{3}=8$ and the only solutions are $i=4,5,6,7,8$, which have, respectively, $\left(p_{2}, p_{3}\right)=(4,0),(3,2),(2,4),(1,6)$ and $(0,8)$. So, 8-hedrites are octahedrites. We will be concerned here only about the generation of $i$-hedrites. Actually, 4-hedrites admit a reasonably simple explicit description, see [DeSt03] and [DeDu08, Chapter 2]. So, it remains to find efficient methods for the enumeration of 5-, 6- and 7-hedrites. The program ENU cannot deal with faces of size 2; so, we sought a method 
TABLE 1 . Number of $i$-hedrites, $4 \leq i \leq 8$, with $2 \leq n \leq 70$

\begin{tabular}{||c|c|c|c|c|c||c|c|c|c|c|c||c|c|c|c|c|c||}
\hline \hline$n$ & $\mathbf{4}$ & $\mathbf{5}$ & $\mathbf{6}$ & $\mathbf{7}$ & $\mathbf{8}$ & $n$ & $\mathbf{4}$ & $\mathbf{5}$ & $\mathbf{6}$ & $\mathbf{7}$ & $\mathbf{8}$ & $n$ & $\mathbf{4}$ & $\mathbf{5}$ & $\mathbf{6}$ & $\mathbf{7}$ & $\mathbf{8}$ \\
\hline \hline 2 & 1 & 0 & 0 & 0 & 0 & 25 & 0 & 12 & 85 & 107 & 51 & 48 & 21 & 45 & 613 & 1574 & 2045 \\
3 & 0 & 1 & 0 & 0 & 0 & 26 & 5 & 16 & 119 & 126 & 109 & 49 & 0 & 40 & 614 & 1751 & 1554 \\
4 & 2 & 0 & 1 & 0 & 0 & 27 & 0 & 21 & 105 & 142 & 78 & 50 & 10 & 54 & 771 & 1874 & 2505 \\
5 & 0 & 1 & 1 & 0 & 0 & 28 & 8 & 18 & 134 & 179 & 144 & 51 & 0 & 66 & 704 & 1963 & 1946 \\
6 & 2 & 2 & 2 & 0 & 1 & 29 & 0 & 16 & 135 & 198 & 106 & 52 & 13 & 58 & 771 & 2247 & 3008 \\
7 & 0 & 3 & 1 & 1 & 0 & 30 & 8 & 24 & 187 & 216 & 218 & 53 & 0 & 48 & 788 & 2419 & 2322 \\
8 & 4 & 1 & 5 & 1 & 1 & 31 & 0 & 32 & 149 & 257 & 150 & 54 & 12 & 66 & 989 & 2511 & 3713 \\
9 & 0 & 2 & 5 & 1 & 1 & 32 & 12 & 24 & 189 & 304 & 274 & 55 & 0 & 92 & 849 & 2735 & 2829 \\
10 & 3 & 3 & 9 & 3 & 2 & 33 & 0 & 18 & 197 & 329 & 212 & 56 & 18 & 68 & 938 & 3041 & 4354 \\
11 & 0 & 5 & 7 & 4 & 1 & 34 & 6 & 26 & 251 & 382 & 382 & 57 & 0 & 49 & 1005 & 3187 & 3418 \\
12 & 5 & 3 & 14 & 5 & 5 & 35 & 0 & 37 & 218 & 431 & 279 & 58 & 9 & 71 & 1175 & 3453 & 5233 \\
13 & 0 & 4 & 14 & 7 & 2 & 36 & 13 & 23 & 278 & 483 & 499 & 59 & 0 & 98 & 1038 & 3659 & 4063 \\
14 & 3 & 7 & 23 & 9 & 8 & 37 & 0 & 24 & 275 & 547 & 366 & 60 & 22 & 70 & 1215 & 3954 & 6234 \\
15 & 0 & 10 & 17 & 12 & 5 & 38 & 6 & 38 & 354 & 601 & 650 & 61 & 0 & 63 & 1193 & 4315 & 4784 \\
16 & 7 & 6 & 28 & 18 & 12 & 39 & 0 & 45 & 313 & 643 & 493 & 62 & 9 & 96 & 1440 & 4526 & 7301 \\
17 & 0 & 6 & 27 & 22 & 8 & 40 & 15 & 37 & 361 & 764 & 815 & 63 & 0 & 104 & 1328 & 4674 & 5740 \\
18 & 5 & 7 & 44 & 25 & 25 & 41 & 0 & 30 & 359 & 838 & 623 & 64 & 21 & 92 & 1378 & 5248 & 8514 \\
19 & 0 & 12 & 35 & 36 & 13 & 42 & 10 & 33 & 472 & 889 & 1083 & 65 & 0 & 74 & 1440 & 5600 & 6631 \\
20 & 7 & 9 & 54 & 46 & 30 & 43 & 0 & 52 & 405 & 998 & 800 & 66 & 14 & 80 & 1751 & 5741 & 10103 \\
21 & 0 & 8 & 57 & 48 & 23 & 44 & 11 & 44 & 480 & 1134 & 1305 & 67 & 0 & 122 & 1531 & 6159 & 7794 \\
22 & 4 & 15 & 77 & 62 & 51 & 45 & 0 & 34 & 511 & 1197 & 1020 & 68 & 16 & 98 & 1675 & 6730 & 11572 \\
23 & 0 & 20 & 59 & 76 & 33 & 46 & 7 & 56 & 609 & 1324 & 1653 & 69 & 0 & 72 & 1792 & 7005 & 9097 \\
24 & 11 & 11 & 87 & 88 & 76 & 47 & 0 & 69 & 519 & 1435 & 1261 & 70 & 14 & 120 & 2066 & 7465 & 13428 \\
\hline \hline
\end{tabular}

that allows for reasonable enumeration of such graphs. See Table 1 for the number of $i$-hedrites with at most 70 vertices. Easy to check that an $n$-vertex $i$-hedrite exists for even $n \geq 2$ if $i=4, n \geq 5$ (and $n=3$ ) if $i=5, n \geq 4$ if $i=6, n \geq 7$ if $i=7, n \geq 8$ (and $n=6$ ) if $i=8$.

Take an $i$-hedrite $G$ with $i \in\{5,6,7\}$. Then, if $F$ is a face of size 2, we reduce it to a vertex by using the following reduction operation:

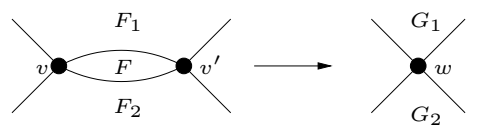

and get a graph denoted by $\operatorname{Red}_{F}(G)$. During this operation the vertices $v$ and $v^{\prime}$ are merged into one vertex $w$ and the faces $F_{1}$ and $F_{2}$ are changed into $G_{1}$ and $G_{2}$ with one edge less. Thus, it is possible that $G_{1}$ and/or $G_{2}$ are themselves of size 2. We apply the reduction operation whenever, by doing it, the reduced graph is still an $i$-hedrite. Eventually, since every application of the technique diminish the vertex-set one obtains a graph, denoted by $\operatorname{Re} d_{\infty}(G)$ for which we cannot apply the reduction operation anymore. 

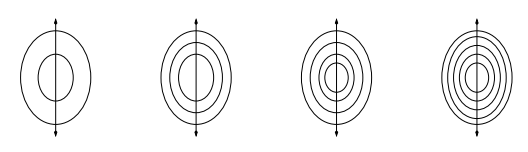

FiguRE 2. Infinite family of unreducible 4-hedrites

We call a graph unreducible if we cannot apply to it any reduction operation. Let $G^{\prime}$ be an unreducible graph. If $G^{\prime}$ has no faces of size 2 , then it is an 8-hedrite, i.e. an octahedrite. If $G^{\prime}$ has a face $F$ of size 2 , then denote by $e_{1}, e_{2}$ the two edges of $F$. Since $G^{\prime}$ is unreducible, $F$ is adjacent on $e_{1}$ or $e_{2}$, say $e_{1}$, to another face of size 2 .

If $e_{2}$ is incident to another face of size 2 , then $G^{\prime}$ is actually $2_{1}$, i.e. the unique graph with two vertices, and four faces of size 2, i.e. the 1 st one on Figure 2, It is easy to see that $e_{2}$ cannot be incident to another face of size 3, but it can be incident to another face of size 4 and in that case $G^{\prime}$ is not 3-connected and thus (see DeDuSh03]) it belongs to the infinite family depicted in Figure 2 .

Call expansion operation the reverse of the reduction operation. The generation method of $i$-hedrite is to consider all unreducible $i$-hedrites and all possible ways of expanding them. For an unreducible graph $G$ denote by $\mathcal{E} x p(G)$ the set of all possible $i$-hedrites that can be obtained by repeated application of the expansion operation. For the graphs of the infinite family of Figure 2 no expansion operation is possible and thus no $i$-hedrite is obtained from them. A priori, the set $\mathcal{E} x p(G)$ can be infinite but, as far as we know, for any 8-hedrite $G$ the set $\mathcal{E} x p(G)$ is finite although we have no proof of it. It turns out that $\mathcal{E} x p\left(2_{1}\right)$ is infinite but it has a simple description.

Theorem 2. (i) The only symmetry groups of 4-hedrites are $D_{4 h}, D_{4}$, $D_{2 h}, D_{2 d}$ and $D_{2}$.

(ii) The only symmetry groups of 5-hedrites are: $D_{3 h}, D_{3}, C_{2 v}, C_{s}$, $C_{2}$ and $C_{1}$.

(iii) The only symmetry groups of 6-hedrites are: $D_{2 d}, D_{2 h}, D_{2}, C_{2 h}$, $C_{2 v}, C_{i}, C_{2}, C_{s}, C_{1}$.

(iv) The only symmetry groups of 7-hedrites are: $C_{2 v}, C_{2}, C_{s}$ and $C_{1}$.

The theorem is proven in the same way as for octahedrites. Minimal representative for each symmetry groups are given in Figures 3, 4, 5, 6.

Further generalization of octahedrites are 4-regular plane graphs with 4-, 3-, 2- and 1-gonal faces only. Then, besides $i$-hedrites, we get graphs with $\left(p_{1}, p_{2}, p_{3}\right)=(2,1,0),(2,0,2),(1,2,1),(1,1,3),(1,0,5)$. 

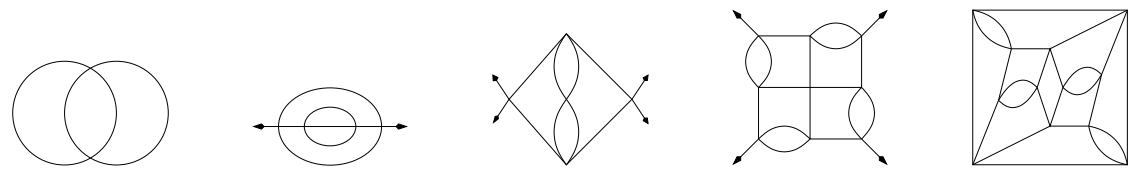

$D_{4 h}, n=2$

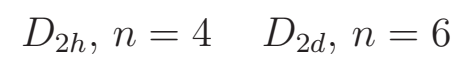

$D_{4}, n=10$

$D_{2}, n=12$

FiguRE 3. Minimal representatives for each possible symmetry group of a 4-hedrite
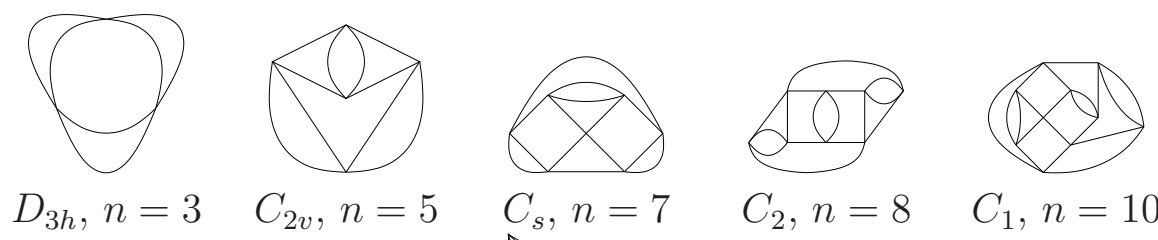

$C_{2}, n=8$

$C_{1}, n=10$

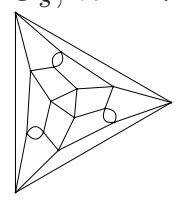

$$
D_{3}, n=15
$$

Figure 4. Minimal representatives for each possible symmetry group of a 5-hedrite
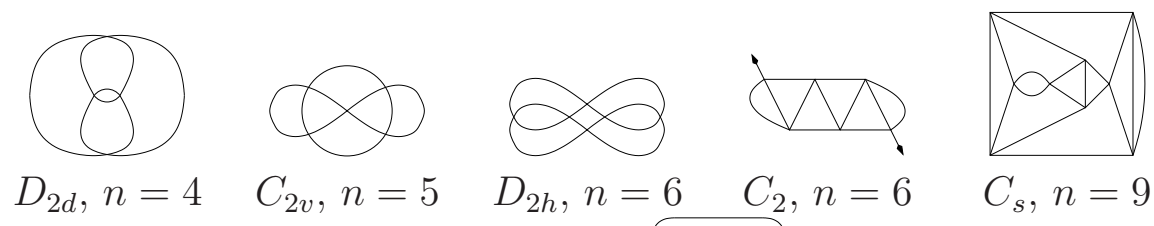

$D_{2 h}, n=6$

$C_{2}, n=6$

$C_{s}, n=9$
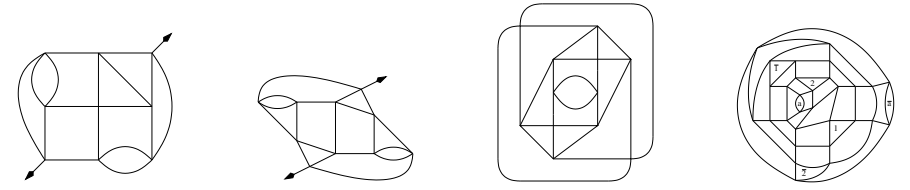

$C_{1}, n=9$

$C_{2 h}, n=10$

$D_{2}, n=12$

$C_{i}, n=30$

Figure 5. Minimal representatives for each possible symmetry group of a 6-hedrite

The enumeration method is then to use $i$-hedrites and to add a 1-gon when we have a pair of 2-gon and 3-gon that are adjacent in all possible ways. This is simlar to the strategy of squeezing of 2-gons used for the enumeration of $i$-hedrites. 


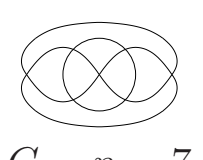

$C_{2 v}, n=7$

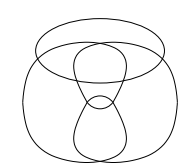

$C_{s}, n=8$

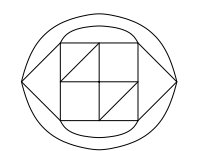

$C_{2}, n=11$

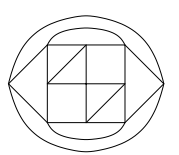

$C_{1}, n=11$

Figure 6. Minimal representatives for each possible symmetry group of a 7 -hedrite

\section{Central circuits and alternating knots}

The edges of an octahedrite, as of any Eulerian plane graph, are partitioned by its central circuits, i.e. those which are obtained by starting with an edge and continuing at each vertex by the edge opposite the entering one. The central circuits of an octahedrite can define circle in the plane or have self-intersections.

If $C_{1}, C_{2}$ are two (possibly, self-intersecting) central circuits of an octahedrite $G$, then they are called parallel if they are separated by a sequence of faces of size 4 (such pair is called railroad in DeDuSh03, DeSt03]). It is possible to reduce those two central circuits into just one and thus get an octahedrite with less vertices. We call an octahedrite irreducible if it has no parallel central circuits. Of course, the reverse operation is possible, i.e. split a central circuit into two or more parallel central circuits. In this way every octahedrite is obtained from an irreducible octahedrite.

It is proved in DeSt03 that an irreducible octahedrite has at most 6 central circuits and in DeDuSh03 that an irreducible $i$-hedrite has at most $i-2$ central circuits. All irreducible octahedrites with non self-intersecting central circuits have been classified in [DeSt03] (see, for another presentation, [DeDuSh03]).

Theorem 3. There are exactly eight irreducible octahedrites with simple central circuits (see Figure 7 ).

A link is a set of circles embedded in 3-space that do not intersect; a link can be represented with its overlapping and underlapping on the plane. A link with only one component is called a knot and Knot Theory is concerned with characterizing different plane presentations of links (see [Li97] for a pleasant introduction). A link is called alternating if it admits a plane representation in which overlappings and underlappings alternate. For a 4-regular plane graph we can define a corresponding alternating link, where the central circuits correspond to 


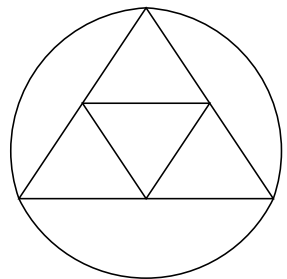

$O_{h}, n=6$

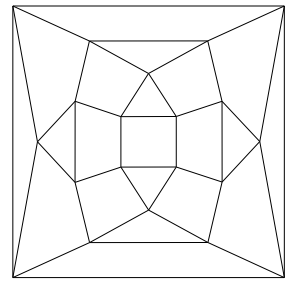

$D_{2 d}, n=20$

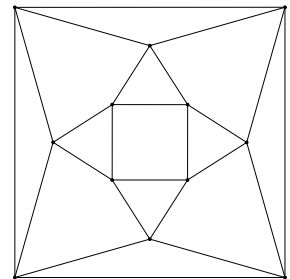

$O_{h}, n=12$

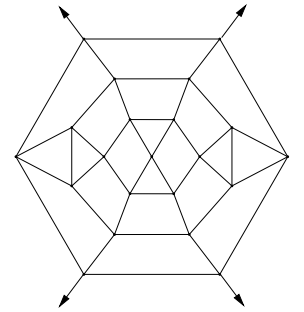

$D_{2 h}, n=22$

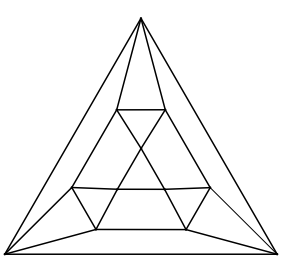

$D_{3 h}, n=12$

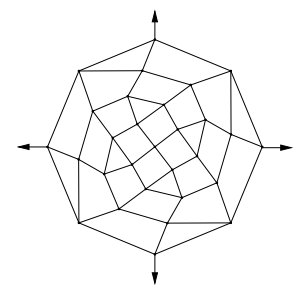

$O, n=30$

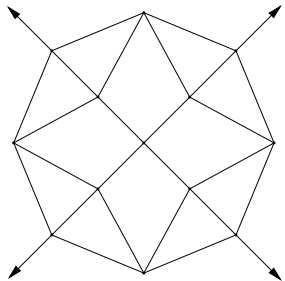

$D_{4 h}, n=14$

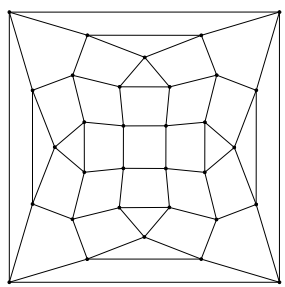

$D_{4 h}, n=32$

FiguRE 7. The irreducible octahedrites with simple central circuits

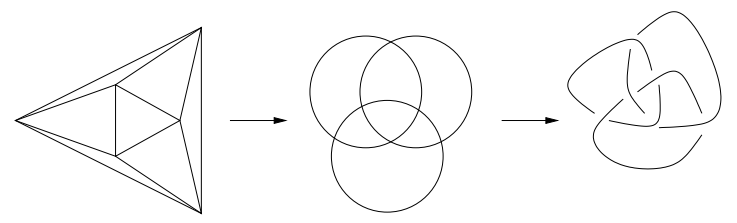

FiguRE 8. The link corresponding to the Octahedron

the components of the link (see an example on Figure 8). It is interesting that there is no known topological characterization of alternating links.

Since an octahedrite with $n$ vertices is 3 -connected, there is no disjointing vertex and thus [Li97, Chapter 5]) the corresponding alternating link cannot be represented with less than $n$ crossings. But it can happen that two octahedrites that are not equivalent as graphs give rise to equivalent alternating links. A link with $m$ components is called Borromean if after removal of any $m-2$ components the remaining two components can be separated one from the other. It is conjectured in DeSt03] that an alternating link obtained from a 4regular 3-connected plane graph is Borromean if and only if for any two central circuits the distance between any two of its consecutive points of its intersection is even. This condition is, of course, sufficient 


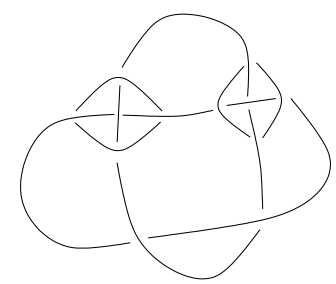

FiguRE 9. A Borromean link

but there are reasons to think that it is not necessary since there exist 4-regular plane graphs (but not 3-connected) which are Borromean without satisfying the specified condition, see Figure 9.

\section{SELF-DUAL GRAPHS}

A graph $G$ is called self-dual if it is isomorphic to its dual $G^{*}$. The medial graph $\operatorname{Med}(G)$ of a plane graph $G$ is the plane graph obtained by putting a vertex on any edge with two edges adjacent if they share a common vertex and are contained in a common face. One has $\operatorname{Med}(G)=\operatorname{Med}\left(G^{*}\right)$. The $\operatorname{graph} G^{\prime}=\operatorname{Med}(G)$ is always 4-regular and its dual $(\operatorname{Med}(G))^{*}$ is bipartite, that is the face-set $\mathcal{F}$ of $\operatorname{Med}(G)$ is split into two sets $\mathcal{F}_{1}\left(G^{\prime}\right)$ and $\mathcal{F}_{2}\left(G^{\prime}\right)$, which correspond to the vertices and faces of the graph $G$. The bipartition $\mathcal{F}_{1}\left(G^{\prime}\right), \mathcal{F}_{2}\left(G^{\prime}\right)$ can be computed easily from a given 4-regular plane graph, i.e. one can compute easily from a graph $G^{\prime}$ the two dual graphs $G_{1}$ and $G_{2}$ such that $G^{\prime}=\operatorname{Med}\left(G_{1}\right)=\operatorname{Med}\left(G_{2}\right)$.

Call $G$ a $i$-self-hedrite if it is a self-dual plane graph with vertices of degree 2,3 or 4 with $v_{2}+v_{3}=i$ and, consequently, faces of size 2,3 or 4. If $G$ is a $i$-self-hedrite then $\operatorname{Med}(G)$ is a $2 i$-hedrite.

The Euler formula $V-E+F=2$ for a self-dual plane graph is, clearly:

$$
\sum_{j=2}^{\infty} p_{j}(4-j)=4 ;
$$

we again permit 2 -gons but not 1 -gons. Define an $i$-self-hedrite to be a such graph with faces of size $2,3,4$ only and $p_{2}+p_{3}=i$. So, $2 p_{2}+p_{3}=4$ and $p_{4}$ is not bounded; also $n=p_{4}+\frac{p_{3}}{2}+2=p_{4}-p_{2}+4$. Clearly, an $i$-self-hedrite can have $i=2,3,4$ only with $\left(p_{2}, p_{3}\right)=(2,0),(1,2),(0,4)$, respectively. The $i$-self-hedrites with smallest number $n$ of vertices have no 4-gons; they are Bundle 2 (2 vertices connected by 2 edges), triangle with one doubled edge and Tetrahedron, respectively. Easy to check that $n$-vertex an $i$-self-hedrite exists if $n \geq i$. 
TABLE 2. Number of $i$-self-hedrites with $4 \leq n \leq 40$ and $2 \leq i \leq 4$

\begin{tabular}{||c|c|c|c||c|c|c|c||c|c|c|c||c|c|c|c||}
\hline \hline$n$ & $\mathbf{2}$ & $\mathbf{3}$ & $\mathbf{4}$ & $n$ & $\mathbf{2}$ & $\mathbf{3}$ & $\mathbf{4}$ & $n$ & $\mathbf{2}$ & $\mathbf{3}$ & $\mathbf{4}$ & $n$ & $\mathbf{2}$ & $\mathbf{3}$ & $\mathbf{4}$ \\
\hline \hline 2 & 1 & 0 & 0 & 12 & 4 & 29 & 24 & 22 & 10 & 90 & 191 & 32 & 9 & 239 & 584 \\
3 & 1 & 1 & 0 & 13 & 6 & 30 & 33 & 23 & 7 & 119 & 198 & 33 & 9 & 256 & 631 \\
4 & 2 & 1 & 1 & 14 & 5 & 42 & 40 & 24 & 7 & 131 & 234 & 34 & 14 & 232 & 748 \\
5 & 2 & 4 & 1 & 15 & 5 & 47 & 48 & 25 & 10 & 124 & 276 & 35 & 10 & 290 & 760 \\
6 & 3 & 6 & 2 & 16 & 8 & 48 & 69 & 26 & 10 & 162 & 304 & 36 & 14 & 308 & 857 \\
7 & 3 & 7 & 4 & 17 & 5 & 64 & 73 & 27 & 8 & 170 & 332 & 37 & 16 & 286 & 956 \\
8 & 3 & 11 & 6 & 18 & 6 & 72 & 92 & 28 & 12 & 158 & 407 & 38 & 11 & 342 & 1002 \\
9 & 3 & 16 & 8 & 19 & 8 & 70 & 114 & 29 & 10 & 190 & 421 & 39 & 11 & 359 & 1070 \\
10 & 5 & 16 & 15 & 20 & 6 & 89 & 130 & 30 & 9 & 210 & 476 & 40 & 16 & 332 & 1239 \\
11 & 4 & 26 & 16 & 21 & 8 & 104 & 148 & 31 & 14 & 202 & 550 & & & & \\
\hline \hline
\end{tabular}

Thus our enumeration method for $i$-self-hedrites is to consider all $2 i$-hedrites $G^{\prime}$, determine for them the graphs $G_{1}, G_{2}$ such that $G^{\prime}=$ $\operatorname{Med}\left(G_{1}\right)=\operatorname{Med}\left(G_{2}\right)$ and keep the ones that have $G_{1}$ isomorphic to $G_{2}$. We denote by $\operatorname{Med}^{-1}\left(G^{\prime}\right)=G_{1} \simeq G_{2}$ the obtained plane graph if it exists. Using the enumeration of $2 i$-hedrites, we can derive the $i$-self-hedrite, see Table 2, Another method would be possible with the results of ArRi92 but it would require more hard programming work and the speed gain is uncertain.

Theorem 4. (i) The possible symmetry groups of 2-self-hedrites graphs are $C_{2}, C_{2 v}, C_{2 h}, D_{2}$ and $D_{2 h}$. Minimal representatives are given in Figure 11 .

(ii) The possible symmetry groups of 3-self-hedrites graphs are $C_{1}$, $C_{2}, C_{s}$ and $C_{2 v}$. Minimal representatives are given in Figure 12.

(iii) The possible symmetry groups of 4-self-hedrites graphs are $C_{1}$, $C_{2}, C_{2 h}, C_{2 \nu}, C_{3}, C_{3 \nu}, C_{4}, C_{4 \nu}, C_{i}, C_{s}, D_{2}, D_{2 d}, D_{2 h}, S_{4}, T, T_{d}$. Minimal representatives are given in Figure 13.

Proof. If $G$ is a 4-self-hedrite then $G^{\prime}=\operatorname{Med}(G)$ is an octahedrite. If $\Gamma, \Gamma^{\prime}$ are the symmetry groups of $G, G^{\prime}$, then the self-duality of $G$ becomes a symmetry in $G^{\prime}$ that exchanges $\mathcal{F}_{1}\left(G^{\prime}\right)$ and $\mathcal{F}_{2}\left(G^{\prime}\right)$. Thus $\Gamma$ is identified with the subgroup of $\Gamma^{\prime}$ formed by the transformations preserving the bipartition. Obviously, the order of $\Gamma$ is half the one of $\Gamma^{\prime}$. The possible groups of $G^{\prime}$ are known (see Theorem 11). So, we set out to enumerate the index 2 subgroups of each of the 18 groups and found, besides the groups in the statement, the groups $D_{3}, D_{4}, C_{4 h}$, $C_{3 h}, S_{6}, S_{8}$ and $T_{h}$. 


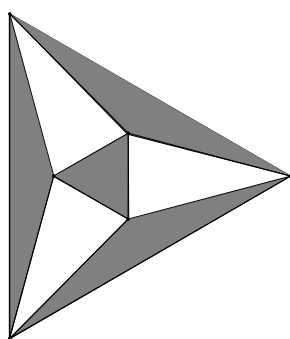

$(k, l)=(1,0)$

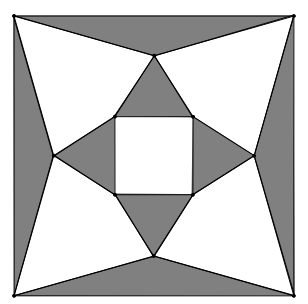

$(k, l)=(1,1)$

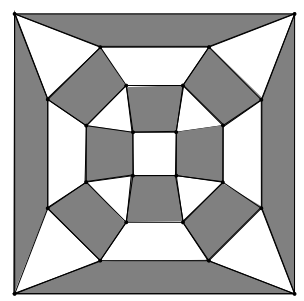

$(k, l)=(2,0)$

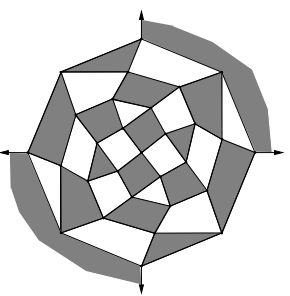

$(k, l)=(2,1)$

Figure 10. First examples of octahedrites of symmetry $O$ or $O_{h}$ expressed as $G C_{k, l}($ Octahedron $)$

The graph $G$ has 4 vertices of degree 3 and 4 faces of size 3 ; both should be partitioned in the same number of orbits and this excludes $D_{4}, D_{3}, C_{3 h}, S_{6}$ and $T_{h}$. Suppose $G$ has symmetry $C_{4 h}$. Due to the plane of symmetry, the 4-fold axis pass through, either two vertices of degree 4 , or through two faces of size 4 . But self-duality requires that it passes through a vertex and a face. The same argument excludes $S_{8}$.

For 2-self hedrites, using the known groups for 4-hedrites gives candidates $C_{2}, C_{2 h}, C_{2 v}, C_{4}, C_{4 h}, C_{4 v}, D_{2}, D_{2 d}, D_{2 h}, D_{4}, S_{4}$. Same kind of orbit reasons exclude $C_{4}, C_{4 h}, C_{4 v}, D_{2 d}, D_{4}, S_{4}$. A 3-self-hedrite has only one vertex of degree 2 that has to be preserved by any symmetry. So, the symmetry is a subgroup of $C_{2 v}$ and all possible subgroups do occur.

It is known DeSt03, DuDe04 that all octahedrites of symmetry $O$ or $O_{h}$ are obtained from the Goldberg-Coxeter construction, i.e. they are of the form $G C_{k, l}$ (Octahedron) for some integer $0 \leq l \leq k$. The pairs $(k, l)$ correspond to the relative position of the triangles; see Figure 10 for the smallest such graphs and DuDe04] for more details on the construction itself.

Theorem 5. All 4-self-hedrites of symmetry $T$ or $T_{d}$ are of the form $\operatorname{Med}^{-1}\left(G C_{k, l}(\right.$ Octahedron $\left.)\right)$ with $k+l$ odd.

Proof. If $G$ is a 4-self-hedrite of symmetry $T$ or $T_{d}$ then its medial $G^{\prime}=\operatorname{Med}(G)$ is an octahedrite of symmetry $O$ or $O_{h}$. So, $G^{\prime}=$ $G C_{k, l}$ (Octahedron) for some $(k, l)$. The automorphism group of the plane graph $G C_{k, l}($ Octahedron) is transitive on triangles; so, we only need to determine when the triangles are not all in $\mathcal{F}_{1}\left(G^{\prime}\right)$ or $\mathcal{F}_{2}\left(G^{\prime}\right)$. Clearly, this correspond to $k+l$ odd.

For a plane graph $G$ a zigzag is a circuit of edges, such that any two but no three, consecutive edges belong to the same face. Zigzags of 


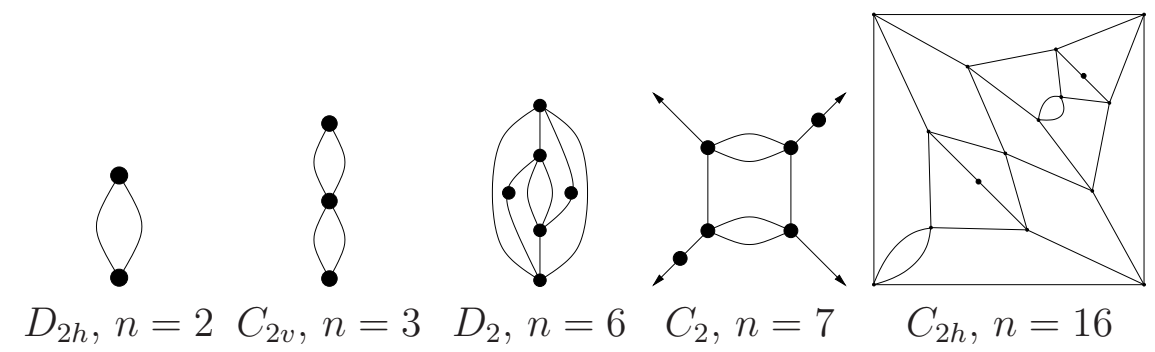

Figure 11. Minimal representatives for each possible symmetry group of 2-self-hedrites

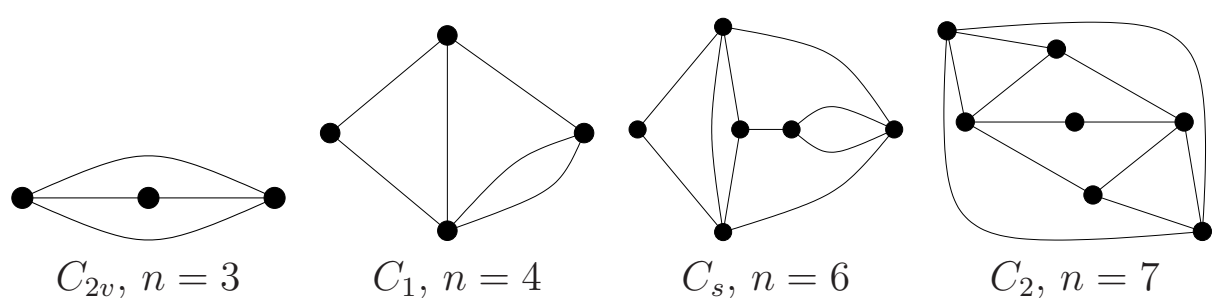

FiguRE 12. Minimal representatives for each possible symmetry group of 3-self-hedrites

$G$ correspond to central circuits of $\operatorname{Med}(G)$, see an example on Figure 14. So, if $G$ is a 4-self-hedrite with simple zigzags, then $\operatorname{Med}(G)$ is an octahedrite with simple central circuits. By Section 4, octahedrites $G^{\prime}$ with simple central circuits are obtained by taking the ones of Figure 7 and splitting each central circuit $C_{i}$ into $m_{i}$ parallel central circuits. Then we have to determine for which $m=\left(m_{i}\right)$ the triangles are in two parts $\mathcal{F}_{1}\left(G^{\prime}\right)$ and $\mathcal{F}_{2}\left(G^{\prime}\right)$ which are equivalent under an automorphism of $G^{\prime}$. This requires a detailed analysis of the automorphism and a search of the necessary relations between $m_{i}$ and parity conditions. The details are very cumbersome but in principle we can get a classification of the 4-self-hedrites with simple zigzags.

In particular, 1, 3, 4, 5, 6, 7th irreducible octahedrites in Figure[7are the medial graphs of 1, 6, 7, 11, 13, 16th 4-self-hedrites in Figure 13, respectively; they are all irreducible 4-self-hedrites with simple zigzags.

\section{Going ON SURFACES}

In DFRR00 was considered a generalization of plane fullerenes on any irreducible surface. Similarly, it is easy to see that any generalized octahedrite, i.e., a 4-regular map on an irreducible surface, having

only 3 - and 4-gonal faces, is either an octahedrite on sphere $S^{2}$, or a 


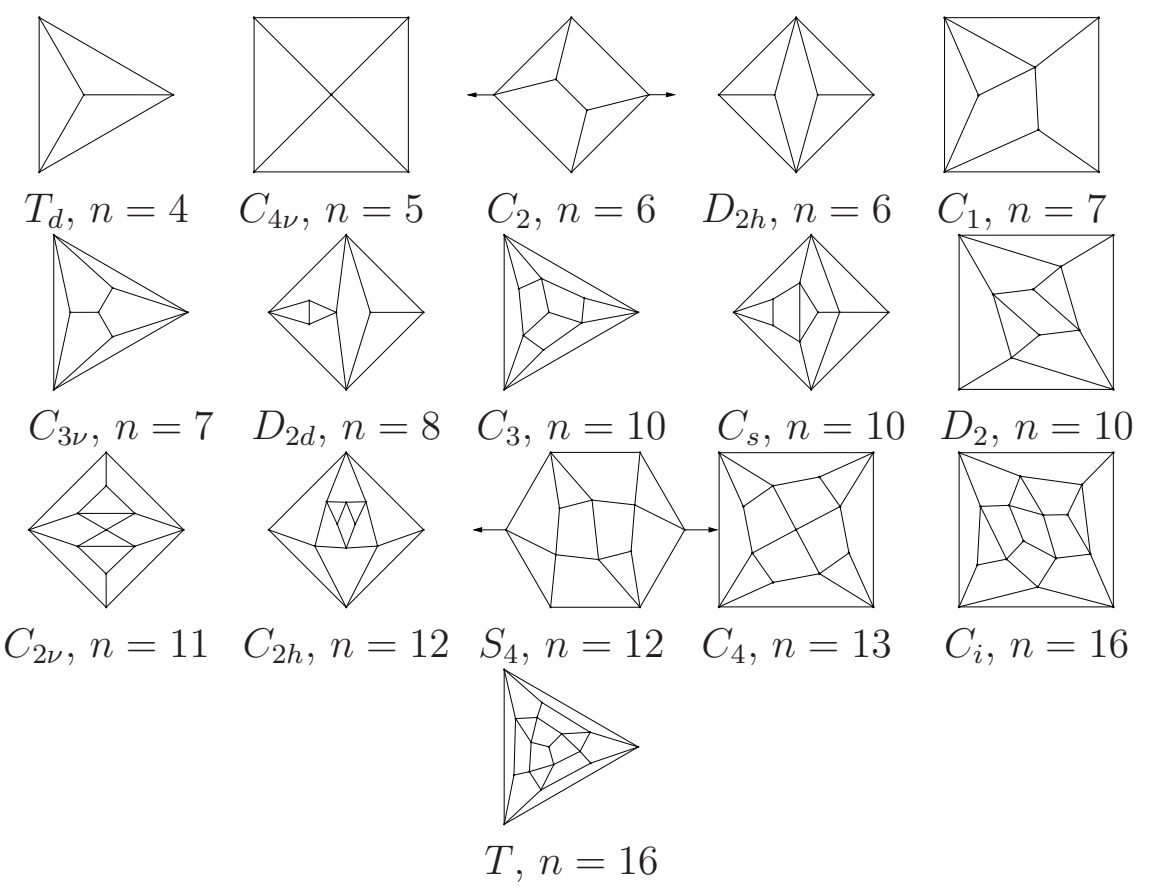

FiguRE 13. Minimal representatives for each possible symmetry group of 4-self-hedrites
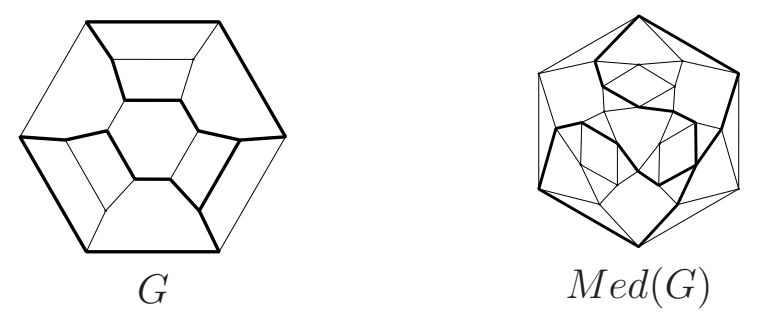

FiguRE 14. Example of a zigzag in a plane graph $G$ and the corresponding central circuit in $\operatorname{Med}(G)$

partition of torus $T^{2}$ (or Klein bottle $K^{2}$ ) into 4-gons, or the antipodal quotient of a centrally symmetric octahedrite on the projective plane $P^{2}$ (having 4 3-gonal faces). Maps on surfaces of high genus can be very complicated. Actually, there are examples with the genus being about half the number of vertices. Here, for the sake of simplicity and the search of more complex examples, we limit ourselves to graphs with no loops or multiple edges. The minimal, i.e. with minimal number of vertices, generalized octahedrite on $S^{2}$ is Octahedron $K_{2,2,2}$; on $P^{2}$ it 
is the antipodal quotient of Cube with two opposite faces triangulated in their center, that is $K_{5}$. On $T^{2}$ it is $K_{5}$, and on $K^{2}$ it is again $K_{2,2,2}$ (but embedded as a quadrangulation); see Fig. 6 in [Na01].

Finally, it is easy to check that any generalized 4-self-hedrite, i.e., self-dual map on an irreducible surface, having only 3- and 4-gonal faces, is either a 4-self-hedrite on sphere $S^{2}$, or a 4-regular partition of torus $T^{2}$ (or Klein bottle $K^{2}$ ) into 4-gons, or the antipodal quotient of a centrally symmetric 4-self-hedrite on the projective plane $P^{2}$ (having 23 -gonal faces). The minimal generalized 4-self-hedrite graph on $S^{2}$ is Tetrahedron; on $P^{2}$ it is the antipodal quotient of the 12th graph on Figure 13, that is complete graph $K_{6}$ with disjoint 2- and 4-vertex paths deleted. On $T^{2}$ it is $K_{5}$, and on $K^{2}$ it is again $K_{2,2,2}$ (see Fig. 6 in [Na01]) embedded as a quadrangulation.

Similar results hold for generalization of $i$-hedrites and $i$-self-hedrites from sphere on any irreducible surface. On $T^{2}$ and $K^{2}$ it gives the 4regular quadrangulations. On $P^{2}$ they are the antipodal quotients of such centrally symmetric graphs on $S^{2}$. So, $2 p_{2}+p_{3}$ becomes 4 for $i$-hedrites and 2 for $i$-self-hedrites on $P^{2}$.

\section{ACKNowledgment}

First author has been supported by the Croatian Ministry of Science, Education and Sport under contract 098-0982705-2707. The authors thank G. Brinkmann for help with the ENU program.

\section{REFERENCES}

[ArRi92] D. Archdeacon and R.B. Richter, The construction and classification of self-dual spherical maps, Journal of Combinatorial Theory B 54 (1992) 37-63.

[BDDH97] G. Brinkmann, O. Delgado-Friedrichs, A. Dress and T. Harmuth, CaGe - a virtual environment for studying some special classes of large molecules, MATCH 36 (1997) 233-237.

[BrHaHe03] G. Brinkmann, T. Harmuth and O. Heidemeier, The construction of cubic and quartic planar maps with prescribed face degrees, Discrete Applied Mathematics, 128-2,3 (2003) 541-554.

[DeDu08] M. Deza and M. Dutour Sikirić, Geometry of chemical graphs, Encyclopedia of Mathematics and its Applications 119, Cambridge University Press, 2008.

[DeDuFo09] M. Deza, M. Dutour Sikiric and P. Fowler, The symmetries of cubic polyhedral graphs with face size no larger than 6, MATCH 61 (2009) 589-602.

[DeDuSh03] M. Deza, M. Dutour and M.I. Shtogrin, 4-valent plane graphs with 2-, 3- and 4-gonal faces, "Advances in Algebra and Related Topics" (in memory of B.H. Neumann; Proc. of ICM Satellite Conference on Algebra and Combinatorics, Hong Kong 2002), World Scientific (2003) 73-97.

[DFRR00] M. Deza, P. W. Fowler, A. Rassat and K. M. Rogers, Fullerenes as Tilings of Surfaces, J. Chem. Inf. Comput. Sci. 40 (2000) 550-558. 
[DeHuLi02] M. Deza, T. Huang and K-W. Lih, Central Circuit Coverings of Octahedrites and Medial Polyhedra, Journal of Math. Research and Exposition 22-1 (2002) 49-66.

[DeSt03] M. Deza and M.I. Shtogrin, Octahedrites, Symmetry 11-1,2,3,4 Special Issue "Polyhedra in Science and Art"(2003) 27-34.

[Du04] M. Dutour, Point Groups, http://www.liga.ens.fr/ dutour/PointGroups/, 2004.

[DuDe04] M. Dutour and M. Deza, Goldberg-Coxeter construction for 3- or 4-valent plane graphs, Electronic Journal of Combinatorics 11 (2004) R20.

[Gr67] B. Grunbaum, Convex Polytopes, Wiley-Interscience, New York, 1967; second edition, Graduate Texts in Mathematics 221, Springer-Verlag, New York, 2003.

[He98] O. Heidemeier, Die Erzeugung von 4-regulären, planaren, simplen, zusammenhängenden Graphen mit vorgegebenen Flächentypen, Diploma thesis, Bielefeld University, Germany, 1998.

[Thu98] W.P. Thurston, Shapes of polyhedra and triangulations of the sphere, in Geometry and Topology Monographs 1, The Epstein Birthday Schrift, ed. by J. Rivin, C. Rourke and C. Series, Geom. Topol. Publ., Coventry (1998) 511-549.

[Li97] W.B. Raymond Lickorish, An Introduction to Knot Theory, Graduate Texts in Mathematics, Springer-Verlag, 1997.

[Ma71] P. Mani, Automorphismen von Polyhedrischen Graphen, Mathematische Annalen 192 (1971) 279-303.

[Na01] A. Nakamoto, Quadrangulations on closed surfaces, Interdisciplinary Information Sciences 7-1 (2001) 77-98.

M. Dutour Sikirić, Rudjer Bosković Institute, Bijenicka 54, 10000

ZaGReB, CROATIA

E-mail address: mdsikir@irb.hr

M. Deza, JAIST, Nomi, Ishikawa-Ken, 923-1292, JAPAN

E-mail address: Michel.Deza@ens.fr 